\title{
Zielsetzung und Methoden der Studie
}

Gesamtüberblick: Das sich aus dem theoretischen Teil ergebene Forschungsinteresse leitet zur Entwicklung eines Instruments zur sprachlichen Variation von Textaufgaben hin, das auf empirisch festgestellten Variationen basiert und spezifische Zielsetzungen verfolgt (Abschnitt 6.1). Unter der Maßgabe der Entwicklung des Instruments ergeben sich ein auf die Zielsetzung ausgerichtetes Studiendesign und eine Auswahl an Methoden, um das Instrument zu konzeptualisieren (Abschnitt 6.2). Unter Rückbeziehung des gewählten Verfahrens auf die in der Theorie gegebenen Ansätze, die die Basis für die empirische Studien bilden, ergeben sich Aspekte, die für die empirische Analyse von sprachlichen Variationen $\mathrm{zu}$ beachten sind (Abschnitt 6.3). Ansatzpunkt des Instruments sind ausgewählte Textmerkmale, die betrachtet und vor der Analyse in einen funktionalen Zusammenhang gebracht werden (Abschnitt 6.4). Die Ermittlung der Textmerkmale erfolgt aus mathematischen Textaufgaben, deren Stichprobenziehung aus Schulbüchern und einem mathematischen Test erfolgt (Abschnitt 6.5).

\subsection{Instrument zur sprachlichen Variation von Textaufgaben}

Aus der in Abschnitt 5.5 erörterten Motivation zur Herstellung eines Instruments, das verwendet werden kann, um sprachliche Textmerkmale in mathematischen Textaufgaben zu verändern, lassen sich grundlegende Elemente ableiten, die das Instrument erfüllen sollte.

Da Mathematikaufgaben (Textaufgaben) ein zentrales Schlüsselelement des Lehrens und Lernens im Mathematikunterricht sind, ist eine evaluierte Möglichkeit der didaktischen Anpassung von Textaufgaben bedeutend. Im Generellen soll

D. Bednorz, Sprachliche Variationen von mathematischen Textaufgaben, Bielefelder Schriften zur Didaktik der Mathematik 5, 
das Instrument dafür verwendet werden, eine Anpassungsstrategie zu bieten, um sowohl in der Forschung als auch in der Praxis den Lernvoraussetzungen und -hindernissen, die im Mathematikunterricht vorkommen, zu begegnen. Ziel ist die vereinfachte Rezeption durch verbesserte Kenntnisse bezüglich der sprachlichen Voraussetzungen und Hindernisse einer Textaufgabe durch ausgewählte sprachliche Varianten von Textaufgaben.

Ein möglicher Ansatzpunkt zur Konzeptualisierung des Modells sind die dimensionalen und interaktionalen Modelle aus der Verständlichkeitsforschung und das in Abschnitt 5.4.2 dargestellte heuristische Modell zur Veränderung der sprachlichen Komplexität bei Mathematikaufgaben. Die Gemeinsamkeit der Verständlichkeitsmodelle und des heuristischen Veränderungsmodells ist, dass die Modelle die Textmerkmale in Gruppen bzw. Dimensionen zusammenfassen und gemeinsam betrachten (Heine et al., 2018; Langer et al., 1974; Groeben, 1982). Die gemeinsame Betrachtung von Textmerkmalen findet in der Betrachtung häufig statt und ist methodisch mit der von Biber (2006) betrachteten Variationsdimensionen in Verbindung zu bringen (vgl. Abschnitt 4.3). Der Unterschied zwischen den Ansätzen liegt insbesondere in der Wahl der Methode, um die Verständlichkeits- bzw. Variationsdimensionen zu bilden. Während Groeben (1982) und Heine et al. (2018) ein empirisch-deduktives Vorgehen wählen, nutzen Langer et al. (1974) und Biber (2006) ein empirisch-induktives Verfahren mit einer Faktorenanalyse, um Verständlichkeits- bzw. Variationsdimensionen zu bilden. ${ }^{1}$ Das empirisch-deduktive Verfahren bietet durch die Festlegung und die Evaluation von relevanten Dimensionen ein elaboriertes Verfahren, um bei der Konstruktion von Mathematikaufgaben beispielsweise für Testsituationen eine Möglichkeit zu besitzen, Anpassungen von Text für den Rezipienten vorzunehmen. Das empirisch-induktive Verfahren bietet den Vorteil, dass die bereits durch Didaktiker oder Schulbuchautoren formulierten Mathematikaufgaben dahingehend analysiert werden können, welche Textmerkmale häufig gemeinsam vorkommen; damit kann die systematische Beziehung der Textmerkmale auf die Faktoren abgebildet werden. Durch die empirische Erhebung der Häufigkeiten und Beziehungen von Textmerkmalen in Textaufgaben, die in der Praxis verwendet werden, wird transparent, welche (natürlichen) sprachlichen Variationen auftreten. Aufgrund der zusätzlichen Möglichkeiten eines empirisch-induktiven Verfahrens erscheint es sinnvoll, ein solches Verfahren auch für Textaufgaben im Mathematikunterricht zu verwenden. Das heißt, dass im sprachlichen Variationsinstrument,

\footnotetext{
${ }^{1}$ Neben der Betrachtung der explorativen Faktorenanalyse von Biber (2006) als Mittel zur Registeranalyse betrachtet Pause (1984) die Faktorenanalyse und die Faktorenbewertung als mögliche Informationsquellen für die Analyse des Textverstehens.
} 
die Variationen von Textmerkmalen, in bereits vorhandenen Textaufgaben in Schulbüchern und Testen als Grundlage betrachtet werden.

Zur Entwicklung des sprachlichen Variationsinstruments unter der erörterten Perspektive ist es notwendig, den Variationsbegriff und den Verständlichkeitsbegriff in die Konstruktion miteinzubeziehen. Dies bedeutet erstens, dass sprachliche Variationen, wie in Abschnitt 4.2 geschildert, als häufiges Auftreten von gewissen Textmerkmalen in einem systematischen Zusammenhang betrachtet werden, und zweitens, dass Textmerkmale ausgewählt werden, die für die in der Mathematik in Abschnitt 4.2 beschriebenen Register typisch oder unter der Perspektive der Textverständlichkeit als relevant zu erachten sind. Drittens bedeutet es, dass die spezifischen sprachlichen Funktionen der Textmerkmale, die diese erfüllen, wie in Abschnitt 4.3 genannt, als Grundlage zur Analyse genutzt werden. Viertens die empirische Erhebung von systematischen Variationen durch die Bildung von Faktoren in der Faktorenanalyse (vgl. Abschnitt 4.5.3 und Abschnitt 5.3.2). Fünftens ist es zentral, durch das Instrument Aussagen darüber treffen zu können, welche Effekte die sprachlichen Variationen von Textmerkmalen auf die Schwierigkeit einer Textaufgabe aufweisen. Damit wird ermöglicht, dass je nach Anpassungsstrategie die sprachlichen Variationen die Schwierigkeit einer Textaufgabe tatsächlich verändern. Sechstens ist es notwendig, den Registerbegriff nicht nur unter der Perspektive der Textmerkmale zu betrachten, sondern auch in Bezug auf kontextbezogene Veränderungen. Das Instrument muss neben der Ebene der sprachlichen Variation ebenfalls die kontextbezogene Variation mitbetrachten und in Beziehung mit Ersterer bringen. Die kontextbezogenen Variationen beziehen sich damit auf den Aufgabentyp der mathematischen Textaufgabe. Dadurch ergibt sich ein robustes Instrument, wodurch Aussagen über die Wahl des Aufgabentyps für die Praxis und für die Testung ermöglicht werden.

Aus den Ausführungen ergeben sich fünf Ziele zur Entwicklung eines empirisch-induktives Instruments zur sprachlichen Variation von Textmerkmalen von mathematischen Textaufgaben:

1. Auswahl geeigneter Textmerkmale

2. Bestimmung der sprachlichen Funktion der Textmerkmale

3. Empirische Feststellung von (sprachlichen) Faktoren (Dimensionen) ${ }^{2}$

\footnotetext{
${ }^{2}$ Zur Vereinfachung der Terminologie wird im empirischen Teil darauf verzichtet, die systematische Gruppierung der Textmerkmale als Dimensionen zu bezeichnen; da die Faktorenanalyse als Verfahren verwendet wird, wird im empirischen Teil ausschließlich die Bezeichnung Faktor/Faktoren verwendet. Im Allgemeinen können die Begriffe Dimensionen und Faktoren in den in der Arbeit gemachten Fokus jedoch als Synonym betrachtet werden.
} 
4. Ermittlung des Effekts auf die Schwierigkeit von mathematischen Testaufgaben

5. Herleitung von kontextbezogenen Spezifika je sprachlichem Faktor

\subsection{Studiendesign und Methode}

Zur Erreichung der in Abschnitt 6.1 geschilderten Ziele ist ein auf sie ausgerichtetes Studiendesign und die passende Wahl der Methoden elementar. Zur quantitativen Beschreibung von Textmerkmalen und für die darauf aufbauende Untersuchung mittels quantitativer Methoden wird ein korpusbasierter Ansatz gewählt (vgl. Abschnitt 4.4). Durch den Korpusansatz werden die in Abschnitt 6.4 ausgewählten Textmerkmale durch eine computerbasierte Ermittlung operationalisierbar.

Um das sprachliche Instrument zur Veränderung zu konzeptualisieren, wird ein Studiendesign gewählt, das auf drei unterschiedlichen Phasen basiert. Der erste Teil der Studie ist sequenziell, während der zweite Teil der Studie als Parallelstudie geplant ist (Kuckartz, 2014). Das Studiendesign entspricht einem Mixed-Methods-Ansatz (Buchholtz, 2019; Kuckartz, 2014). In Abbildung 6.1 ist das gewählte Studiendesign schematisch dargestellt.

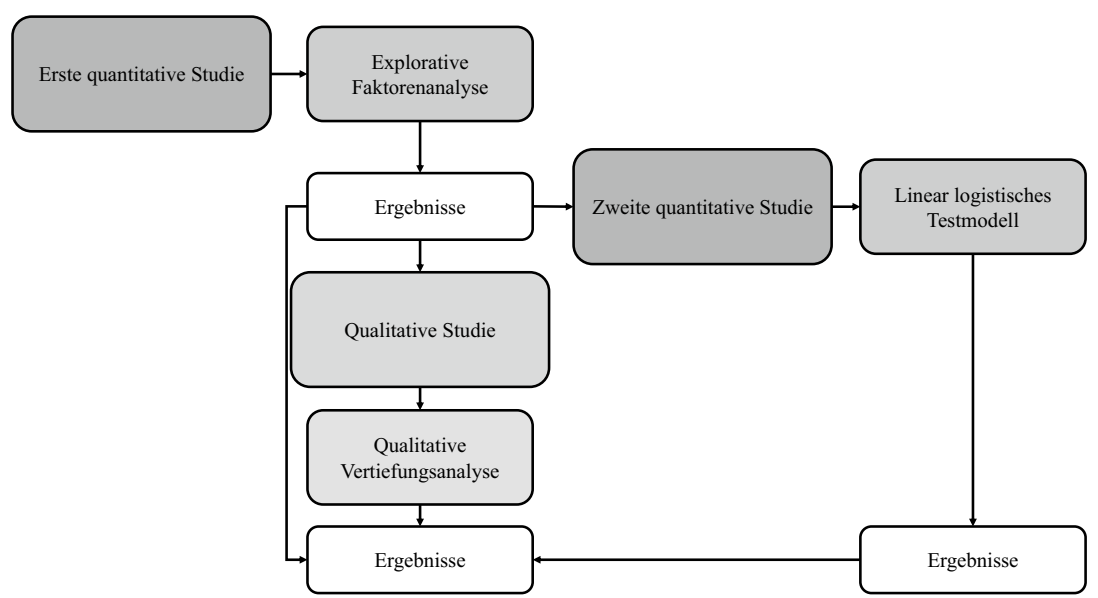

Abbildung 6.1 Drei-Phasen-Design. (Eigene Erstellung) 
Die erste quantitative Studie forciert die Erhebung der ausgewählten Textmerkmale aus einer in Abschnitt 6.5 erläuterten Stichprobenziehung von mathematischen Textaufgaben und die Operationalisierung durch den angesprochenen korpusbasierten Ansatz. Hauptanalyseteil ist die anschließende explorative Faktorenanalyse, um die systematischen Textvariationen in den ausgewählten mathematischen Textaufgaben zu bestimmen. Die erste quantitative Studie verfolgt damit die in Abschnitt 6.1 definierten ersten drei Zielsetzungen. Der anschließende Teil der Parallelstudie besteht zum einen aus einer zweiten quantitativen Studie und zum anderen aus einer qualitativen Studie. Der zweite quantitative Teil verwendet die Ergebnisse der Faktorenanalyse, um die vierte Zielsetzung in Abschnitt 6.1 zu beantworten. Durch die Faktorenanalyse kann für jede Textaufgabe im Datensatz ein Faktorenwert ermittelt werden. Der Faktorenwert repräsentiert, wie gering oder hoch die Beziehung zwischen Textaufgabe und Faktor ist. Dieser Faktorwert kann als Aufgabenmerkmal genutzt werden, um den Effekt auf die Schwierigkeit einer mathematischen Textaufgabe zu berechnen. Der Effekt auf die Aufgabenschwierigkeit wird durch die Faktorenwerte (eines Teils) der Textaufgaben (vgl. Abschnitt 6.5) in einem linear-logistischen Testmodell berechnet. Sowohl Cluster- als auch Regressionsanalysen dienen zur Spezifizierung des Effekts. Der dritte Teil der Gesamtstudie bezieht sich auf die fünfte Zielsetzung in Abschnitt 6.1. Grundlage sind wiederum die Faktorenwerte der Textaufgaben. Aus dem Gesamtdatensatz werden diejenigen Textaufgaben betrachtet, die besonders hohe Faktorenwerte für einzelne Faktoren zeigen, also repräsentativ für diesen Faktor und weniger repräsentativ für die restlichen Faktoren sind. Durch die Auswahl repräsentativer Textaufgaben können kontextuelle Spezifika dieser Textaufgaben herauskristallisiert werden. Dies geschieht durch eine qualitative Vertiefungsanalyse, einem deduktiven und induktiven Vorgehen zur Bildung eines Kategoriensystem, das genutzt werden kann, um die repräsentativen Textaufgaben je Faktor zu analysieren und Aufgabentypen herauszubilden.

\subsection{Hinweise zur empirischen Analyse}

Aufgrund der Anlehnung der Interpretation der Faktoren an Biber (2006) und seiner Konzeption von sprachlichen Variationsdimensionen wird nachfolgend auf die genannten Maßgaben der Voraussetzungen für Studien, die sprachliche Dimensionen analysieren, eingegangen.

Um eine ausreichende Analyse der Variationen in einer Domäne zu untersuchen, benötigt es eine angemessene und repräsentative Stichprobe, sowohl der 
untersuchten Textmerkmale als Variablen als auch der verwendeten Textstichprobe, die als Korpus dient.

Nach Biber (2006) sind drei Kriterien zur Abbildung eines umfassenden analytischen Rahmens besonders relevant:

1. Die Analyse soll alle bedeutenden linguistischen Merkmale eines Registers beinhalten, einschließlich der möglichen Zusammenhangsbeziehungen zwischen den Merkmalen.

2. Es soll ein vollständiges Abbild der situationsbedingten Charakterisierung einzelner Register sowie die Spezifikation der Ähnlichkeiten und Unterschiede zwischen den sprachlichen Variationen möglich sein.

3. Die Analyse sollte einen formalen Rahmen darbieten, um die Beziehungen festzustellen, die durch die Verwendung von multivariaten (multidimensionalen) Ansätzen durch die Methode selbst geleistet werden.

Bezüglich der ersten und zweiten Voraussetzung ist darauf zu achten, dass für die Analyse eine systematische Stichprobenziehung (vgl. Abschnitt 6.5) von Texten erfolgt und dass die verwendeten Textmerkmale die Varianz an vorhandenen Textmerkmalen und möglichen Zusammenhangsbeziehungen ausreichend abdecken. Biber und Egbert (2018) unterscheiden für die Verwendung des multivariaten Verfahrens ebenfalls drei Stufen:

1. Die Bestimmung der Ausprägung der Textmerkmale je Aufgabe.

2. Die Verwendung der Faktorenanalyse, um die Faktoren festzustellen.

3. Die funktionale Interpretation der Faktoren.

Die Faktoren der Faktorenanalyse können sowohl auf Ebene der Vertextung als auch auf funktionaler Basis beschrieben werden. Die sprachliche Ebene ist durch die Gruppe von Textmerkmalen bestimmt, die auf einem Faktor besonders repräsentiert wird (vgl. Abschnitt 7.2.4). Nach Biber und Egbert (2018) kann auf funktionaler Basis der Faktor durch die funktionalen Merkmale interpretiert werden, die die Textmerkmale am häufigsten teilen. Für die funktionale Interpretation wird angenommen, dass die systematischen Variationen die zugrundeliegenden Kommunikationsfunktionen widerspiegeln. Auf Basis der Identifikation der Faktoren werden über die Interpretation der spezifischen Textmerkmalsvariation je Faktor Bezeichnungen gewählt, die der Kommunikationsfunktion entsprechen. Die Herstellung und Interpretation der Variationen der Faktoren bietet die Möglichkeit, zu bestimmen, welche besonderen sprachlichen Voraussetzungen für die kommunikativen Funktionen erfüllt werden müssen. Für die Interpretation der 
Faktoren ist der Einbezug der Ähnlichkeiten und Unterschiede zwischen den Faktoren zentral. Für den Vergleich der Dimensionsinterpretation schlagen Biber und Egbert (2018) die Bildung von Faktorenbewertungen anhand der Faktorenwerte vor. In Anlehnung daran wird in Abschnitt 7.2.5 eine Bewertung gebildet und für die Analyse verwendet.

Eine besondere Schwierigkeit bei der Analyse von Textmerkmalen und der Nutzung des Registerbegriffs ist die explizite Zuordnung zwischen Merkmalsträgern und Ausprägungen als Register. Diese diskrete Zuordnung bzw. Trennung ist für die Analyse schwierig, da die unterschiedlichen Register gleiche Textmerkmale verwenden, jedoch gegebenenfalls unterschiedlich frequentiert oder in einer anderen Bedeutung. Aus diesem Grund verwendet diese Arbeit dieselbe Annahme wie Biber (2006), der die sprachlichen Variationen und die Registerunterschiede als einen kontinuierlichen Variationsraum definiert, für den kein Versuch unternommen wird, eine diskrete Ebene von Registern zu bestimmen. Register werden entsprechend als kontinuierliches und nichtdiskretes Konstrukt betrachtet.

\subsection{Auswahl der Textmerkmale für die Analyse und funktionale Gruppierung}

Die Analyse von Textmerkmalen durch ein korpusbasiertes Vorgehen ist aufwendig. Aufgrund von begrenzten Ressourcen muss eine Abwägung getroffen werden, welche Textmerkmale für die Analyse betrachtet werden. Insgesamt wurden 17 Textmerkmale ausgewählt, die unter der Maßgabe der Erläuterungen in Kapitel 4 und 5 als relevante Textmerkmale für mathematische Textaufgaben betrachtet werden können und unterschiedliche funktionale Aspekte im Text erfüllen. Die ausgewählten Textmerkmale werden nachfolgend beschrieben, während die Erklärung der Kodierung der Textmerkmale in den Abschnitten 7.1.2 und 7.1.3 erfolgt.

- Mathematische Begriffe: Mathematische Begriffe sind die Grundlage der Kommunikation von mathematischen Objekten und Operationen und haben eine hohe Bedeutung für den Aspekt Sprache als Lerngegenstand im Mathematikunterricht.

- Diskontinuierlicher Text: Mathematische Textaufgaben können durch Abbildungen, Tabellen oder Illustrationen unterbrochen werden. Sie können als Hilfe 
dienen, aber auch als Lerngegenstand und aufgrund der typischen Text-BildReferenzen in der mathematischen Kommunikation sind sie ein bedeutender Bestandteil von Textaufgaben.

- Symbole: Mathematische Kommunikation verfolgt eine präzise Darstellung von mathematischen Objekten und Operationen. Symbole ermöglichen eine Verdichtung der Kommunikation durch Formalisierung und die Reduktion von Redundanz. Symbole sind typische Merkmale von mathematikhaltigen Texten.

- Zahlen: Zahlen als mathematische Objekte sind grundlegend für viele mathematische Textaufgaben zur Berechnung von ausgewählten Sachverhalten. Durch Zahlen werden explizit Anzahl und Größen repräsentiert.

- Nominalisierung: Die Objektivierung von Verben in Substantiven ist typisch für bildungssprachliche und fachsprachliche Kommunikation. Durch die Verwendung von Nominalisierungen wird die Kommunikation verdichtet, da nicht Handlungen, sondern Objekte beschrieben werden.

- Durchschnittliche Silbenanzahl im Text: Fachsprachliche Texte haben häufig einen hohen Anteil an komplexen Wörtern. Komplexe Wörter zeichnen sich unter anderem durch eine hohe Anzahl an Silben aus, die Informationen und Aussagen verdichten. Die durchschnittliche Silbenanzahl im Text kann als Indikator für die Komplexität (durch die Anzahl der Silben) der Wörter in einem Text betrachtet werden.

- Gebräuchlichkeit des Wortschatzes im Text: Fachsprachliche Texte zeichnen sich, wie beim Textmerkmal durchschnittliche Silbenanzahl beschrieben, durch einen hohen Anteil an komplexen Wörtern aus. Ein zweiter Indikator, der Aufschluss über die Komplexität der Wörter geben kann, ist die Frequenz der Wörter im Wortschatz. Häufige Wörter sind leichter, seltene Wörter kompliziert. Durch den Indikator Gebräuchlichkeit des Wortschatzes wird die Häufigkeit der Einzelwörter in einem Referenzkorpus ermittelt und für den Text berechnet. Ist die Gebräuchlichkeit hoch, ist davon auszugehen, dass Inhalte, Gegenstände und Informationen explizit (deutlich bzw. klar) dargestellt werden sollen.

- Passiv: Die Formulierung im Passiv ist ein typisches stilistisches Textmittel der bildungs- und fachsprachlichen Verwendung. Die Verwendung des Passivs dient insbesondere der Verallgemeinerung von Sätzen.

- Unpersönliche Sprache: Die Vermeidung von Eigennamen und Personalpronomen kann zur Verwendung von unpersönlichen sprachlichen Mitteln führen, 
beispielsweise von Man- und Es-Konstruktionen. Solche Konstruktionen dienen ebenfalls der Verallgemeinerung von Sätzen.

- Füllwörter: Füllwörter sind typisch für die mündliche Kommunikation, wird jedoch auch für schriftliche Kommunikation verwendet, um zusätzliche Redundanz zu schaffen. Daneben erscheinen Texte mit Füllworten weniger verallgemeinernd, z. B.: ,muss es auch ...“

- Modalverben: Modalverben dienen dazu, Handlungen als Möglichkeiten oder Notwendigkeiten zu formulieren. Durch die Verwendung ergeben sich Charakteristika von Diskussionsstrukturen in einem Text: „Muss es so sein?“, „Wollen etwas kaufen!“

- Verben im Perfekt: Die Verwendung von Verben im Perfekt ist für mathematische Textaufgaben häufig, da Situationen dargestellt werden, die zwar abgeschlossen sind, deren Wirkung sich jedoch noch auf die Gegenwart ausprägt und die Behandlung der Aufgabe durch den Lernenden motiviert.

- Lexikalische Vielfalt: Die Verwendung von unterschiedlichen Wörtern in einem Text ist typisch für bildungssprachliche und fachsprachliche Texte. Das liegt an der Verwendung von vielen Termini in bildungssprachlichen und fachsprachlichen Texten und an der Stilistik, direkte Wortwiederholung zu vermeiden.

- Propositionaler Gehalt: Bildungs- und fachsprachliche Texte zeichnen sich durch eine hohe Informationsdichte aus. Propositionen bilden die Informationsdichte ab. Der Indikator propositionaler Gehalt betrachtet die Anzahl der Propositionen im Verhältnis zu den vorhandenen Sätzen im Text und gibt im Mittel an, wie viele Propositionen in einem Satz verwendet werden.

- Direkte Anaphorik: Direkte Bezüge zu bereits verwendeten Begriffen erzeugen eine hohe Kohärenz in einem Text, indem die Referenzen im Text klar sind. In mathematischen Textaufgaben kann die Anzahl der Verwendungen von direkter Anaphorik als Maß dafür angesehen werden, wie deutlich Referenzen im Text gekennzeichnet werden.

- Konjunktionen: Die Verknüpfung von Textteilen kann durch Konjunktionen geschehen. Konjunktionen sind ein bedeutender Bestandteil, um logische Verknüpfungen herzustellen. 
- Präpositionen: Präpositionen sind ein bedeutendes sprachliches Mittel für mathematische Texte. Sie ermöglichen die Kommunikation von Raum (Lokalität) und Zeit (Temporalität), die für die Vermittlung von Real- und Formalbezügen in Textaufgaben zentral sind.

Als Hilfestellung zur Interpretation dient die aus der Literatur und in Kapitel 4 dargestellte Verbindung zwischen Textmerkmalen (Brinker, 2010; Feilke, 2012b; Gogolin, Neumann und Roth, 2007; Halliday, 2014a). Die Einteilungen der Tabellen 6.1 bis 6.3 sind an die Zuordnung von Feilke (2012b) angelehnt. Die nach Feilke (2012b) gemachte Zuordnung wurde durch den in Tabelle 6.3 abgebildeten Textaspekt ergänzt. Die Ergänzung orientiert sich dabei insbesondere an der in Abschnitt 2.3.3 diskutierten funktionalen Unterscheidung von Sprache durch Halliday (2014a). In Anbetracht der von Feilke (2012b) verwendeten Begriffe Inhaltsaspekt und Beziehungsaspekt kann eine inhaltliche Nähe mit der von Halliday (2014a) betrachteten ideellen und interpersonalen Metafunktion hergestellt werden, die grundlegend dieselben zwei funktionalen Beschreibungen von Sprache definiert. In Hinblick auf Hallidays (2014a) textuelle Metafunktion wird diese Kategorie für die Zuordnungen ergänzt und in Anlehnung an die Begrifflichkeiten nach Feilke (2012b) als Textaspekt bezeichnet.

In Tabelle 6.1 sind die gemachten Zuordnungen zum Inhaltsaspekt zu erkennen. Der Inhaltsaspekt, der die Aussageinformation forciert, ist durch zwei verschiedene Sprecherstrategien gekennzeichnet. Die erste Sprecherstrategie ist das Explizieren und damit das Verdeutlichen eines Gegenstands oder Begriffs. Da es sich bei den Gegenständen und Objekten im Mathematikunterricht um mathematische Objekte handelt, wurden die Textmerkmale mathematische Begriffe und Zahlen dieser Kategorie zugeordnet.

Tabelle 6.1 Funktionale Zuordnung der sprachlichen Merkmale zur Interpretation inhaltlich-funktionaler Aspekte

\begin{tabular}{l|l|l}
\hline $\begin{array}{l}\text { Sprachliche } \\
\text { Funktion }\end{array}$ & Sprecher-Strategie & Textmerkmale \\
\hline Inhaltsaspekt & Explizieren & $\begin{array}{l}\text { Mathematische } \\
\text { Begriffe }\end{array}$ \\
\cline { 3 - 3 } & & Zahlen \\
& $\begin{array}{l}\text { Gebräuchlichkeit } \\
\text { des Wortschatzes }\end{array}$ \\
\cline { 2 - 3 } & Verdichten & $\begin{array}{l}\text { Propositionaler } \\
\text { Gehalt }\end{array}$ \\
\hline
\end{tabular}

(Fortsetzung) 
Tabelle 6.1 (Fortsetzung)

\begin{tabular}{l|l|l}
\hline $\begin{array}{l}\text { Sprachliche } \\
\text { Funktion }\end{array}$ & Sprecher-Strategie & Textmerkmale \\
\hline & & $\varnothing$ Silbenanzahl \\
\cline { 3 - 3 } & & Symbole \\
& & Nominalisierung \\
\hline
\end{tabular}

Des Weiteren wurde das Textmerkmal Gebräuchlichkeit des Wortschatzes der Strategie des Explizierens zugeordnet. Eine hohe Gebräuchlichkeit des Wortschatzes weist auf eine nichtfachspezifische Explikation von Informationen, Handlungen und Gegenständen hin. Die zweite Sprecherstrategie ist das Verdichten, das komprimierende Strategien zusammenfasst. Entsprechend wurden die Variablen propositionaler Gehalt, durchschnittliche Silbenanzahl, Symbole und Nominalisierung diesen Aspekt zugeordnet. Die unterschiedlichen Variablen haben eine verdichtende Wirkung, entweder durch Steigerung der Aussagedichte des Textes (propositionaler Gehalt) oder durch Verdichtung auf der Ebene von Wörtern (durchschnittliche Silbenanzahl, Symbole und Nominalisierung).

Tabelle 6.2 Funktionale Zuordnung der sprachlichen Merkmale zur Interpretation interpersonaler Aspekte

\begin{tabular}{l|l|l}
\hline $\begin{array}{l}\text { Sprachliche } \\
\text { Funktion }\end{array}$ & Sprecher-Strategie & Textmerkmale \\
\hline Beziehungsaspekt & Verallgemeinern & Passiv \\
\cline { 3 - 3 } & & $\begin{array}{l}\text { Unpersönliche } \\
\text { Sprache }\end{array}$ \\
\cline { 2 - 3 } & Diskutieren & Füllwörter \\
\cline { 3 - 3 } & & Modalverben \\
\hline
\end{tabular}

In Tabelle 6.2 sind die Einteilungen bezüglich des Beziehungsaspekts dargestellt, die die Sprecherabsicht fokussieren. Für diese sprachliche Funktion wurden die Strategien Verallgemeinern und Diskutieren zugeordnet. Das Verallgemeinern, also Gegenstände und Sachverhalte durch sprachliche Mittel zu abstrahieren, wird durch die sprachliche Variable Passiv abgebildet, da Texte tendenziell durch das Passiv abstrahiert werden, beispielsweise indem das Subjekt in Passivsatzstrukturen weggelassen wird. Außerdem wird das Textmerkmal unpersönliche Sprache dem Verallgemeinern zugeordnet. Das Diskutieren, also die sprachliche Äußerung als offene Aussage zu betrachten, wird mit den Textmerkmalen Füllwörter aufgrund von Relativierungen (auch, manchmal) und Modalverben (können, wollen) 
in Beziehung gesetzt. Außerdem ist das Textmerkmal Perfekt tendenziell dem Diskutieren zugeordnet, da im Perfekt ein Verb verwendet wird, um Handlungen auszudrücken, die vollendet sind, die sich jedoch noch auf die Gegenwart beziehen. Damit können Handlungen vermittelt werden, die begangenen worden sind und über deren Urteil noch zu entscheiden bzw. zu diskutieren sind.

Tabelle 6.3 Funktionale Zuordnung der sprachlichen Merkmale zur Interpretation textueller Aspekte

\begin{tabular}{l|l|l}
\hline Sprachliche Funktion & Sprecher-Strategie & Textmerkmale \\
\hline Textaspekt & Relator & Konjunktionen \\
\cline { 2 - 3 } & & Präpositionen \\
\cline { 2 - 3 } & Referenz & Diskontinuierlicher Text \\
& & Direkte Anaphorik \\
& & Lexikalische Vielfalt \\
\hline
\end{tabular}

In Tabelle 6.3 sind die letzten Einteilungen zu erkennen. Die sprachliche Funktion des Textaspekts beschreibt insbesondere die Bildung von Kohäsion in einem Text. Zum einen wird der Textaspekt bezogen auf die Sprecherstrategie durch Relatoren ermöglicht. Die zentralen Textmerkmale für Relatoren sind Konjunktionen, die als Bindewörter und Präpositionen als Verhältniswörter die Kohäsion in einem Text ermöglichen. Zum anderen sind Referenzen innerhalb des Textes für den Textaspekt bedeutend. Zu Referenzen werden drei Textmerkmale gezählt: zum einen diskontinuierlicher Text, der die Referenz zwischen Bild und Text umfasst, und zum anderen direkte Anaphorik, die die Referenz zwischen Sätzen ermöglicht. Drittens wird das Textmerkmal lexikalische Vielfalt den Referenzen zugeordnet, da die Höhe der lexikalischen Vielfalt Aussagen über die mögliche Anzahl an direkten Kohäsionsstrukturen ermöglicht. So ist beispielsweise die lexikalische Vielfalt geringer, wenn in einem Text der Name Kevin in jedem Satz wiederaufgenommen und nicht gegen ein Personalpronomen ausgetauscht wird, beispielsweise $e r$.

\subsection{Stichprobe}

Für die Auswahl der Textstichprobe, die als Korpus für die Analyse dient, wurde eine systematische Stichprobenziehung durchgeführt. Dafür wurden aus einer Auswahl von 30 Schulbüchern aus Nordrhein-Westfalen (NRW) zufällig neun 
Schulbücher gezogen. Aus den Schulbüchern wurden wiederum zwischen 30-40 Textaufgaben, je nach Passung der vorhandenen Textaufgaben, gezogen. Für die Anzahl der gewählten Textaufgaben wurde insbesondere die Stichprobengröße, die für die Verwendung in einer Faktorenanalyse zu beachten ist, berücksichtigt (Bortz und Schuster, 2010). Es ergaben sich insgesamt $N=295$ Textaufgaben aus den Schulbüchern. Für die Erfüllung der in Abschnitt 6.1 formulierten vierten Zielsetzung wurden zusätzlich 47 Textaufgaben im offenen Aufgabenformat aus der PALMA-Studie ausgewählt (vgl. Kapitel 8; Pekrun et al., 2006; vom Hofe et al., 2002). Damit ergab sich für den Korpus, der für die Analyse genutzt wurde, eine Aufgabenanzahl von $N=342$ Textaufgaben. Dabei konnten $58.57 \%$ dem Inhaltsbereich Algebra, $25.58 \%$ den Inhaltsbereich Geometrie und $15.85 \%$ dem Inhaltsbereich Stochastik zugeordnet werden.

\subsection{Zusammenfassung}

Aus dem Forschungsinteresse ergibt sich die Formulierung spezifischer Kriterien für die Entwicklung des Instruments zur sprachlichen Variation von Textaufgaben. Das führt zur Zielsetzung von fünf Aspekten, die das Instrument erfüllen soll. Aus den Zielsetzungen für die Konzeptualisierung des Instruments ergibt sich ein Studiendesign, das auf drei Phasen basiert, zwei quantitativen und einer qualitativen Studie, die unterschiedliche Methoden verwenden, um die Zielsetzungen zu erfüllen. Für das empirische Vorgehen werden aufgrund der Anlehnung an bereits etablierte Analysen Voraussetzungen und Annahmen getroffen, die die Vorgehensweise prägen. Für die Analyse wurden 17 Textmerkmale ausgewählt, die als besonders relevant für die Untersuchung von mathematischen Textaufgaben betrachtet und in einen funktionalen Zusammenhang gebracht werden können. Die für den Korpus verwendeten Textaufgaben wurden systematisch aus neun Schulbüchern und ergänzend aus bereits durchgeführten mathematischen Tests gezogen.

Ausblick: Nach der Beschreibung der Anlage der Arbeit wird anschließend die erste quantitative Studie beschrieben. Hierbei wird aufgrund der unterschiedlichen Auswertungsmethoden der Einzelstudien auf die Auswertungsgrundlage der Faktorenanalyse eingegangen und anschließend das Ergebnis der Faktorenanalyse präsentiert. 
Open Access Dieses Kapitel wird unter der Creative Commons Namensnennung 4.0 International Lizenz (http://creativecommons.org/licenses/by/4.0/deed.de) veröffentlicht, welche die Nutzung, Vervielfältigung, Bearbeitung, Verbreitung und Wiedergabe in jeglichem Medium und Format erlaubt, sofern Sie den/die ursprünglichen Autor(en) und die Quelle ordnungsgemäß nennen, einen Link zur Creative Commons Lizenz beifügen und angeben, ob Änderungen vorgenommen wurden.

Die in diesem Kapitel enthaltenen Bilder und sonstiges Drittmaterial unterliegen ebenfalls der genannten Creative Commons Lizenz, sofern sich aus der Abbildungslegende nichts anderes ergibt. Sofern das betreffende Material nicht unter der genannten Creative Commons Lizenz steht und die betreffende Handlung nicht nach gesetzlichen Vorschriften erlaubt ist, ist für die oben aufgeführten Weiterverwendungen des Materials die Einwilligung des jeweiligen Rechteinhabers einzuholen. 